\title{
Cycle de l'oogénèse chez les femelles de Glossina tachinoides West. et détermination de l'âge physiologique
}

\author{
par J. ITARD
}

\begin{abstract}
RÉSUMÉ
A partir de femelles de Gl. tachinoides West. élevées au laboratoire à $25^{\circ} \mathrm{C}$ et 70 p. 100 d'H. R. et fécondées à l'âge de 3 jours. une étude de l'appareil génital et du cycle de l'oogénèse o été entreprise chez cette espèce.

L'anatomie et la morphologie des organes reproducteurs des femelles de cette espèce ne diffèrent que très peu de celles déjà décrites chez d'autres espèces du même genre.

Le cycle de l'oogénèse est également sensiblement identique à celui des espèces déjà étudiées par différents auteurs.

Une méthode et un tablequ de détermination de l'âge physiologique des femelles de Gl. tachinoides sont proposés. La méthode décrite permet d'évaluer l'âge d'une femelle jusqu'au $80^{\circ}$ jour environ.
\end{abstract}

Une connaissance approfondie de la biologie des insectes vecteurs est nécessaire pour comprendre l'épidémiologie des maladies transmises et adapter à la situation locale les moyens ef les techniques de lutte. La possibilité de déterminer l'âge d'un insecte est précieuse pour connaître la biologie de l'espèce. Les méthodes de diagnose de l'âge physiologique chez les femelles de moustiques ont été décrites par DETINOVA (1963). Les méthodes les plus précises sont basées sur les modifications que subit l'appareil reproducteur de la femelle au cours de sa vie. DETINOVA a également décrit les modifications survenant chez un certain nombre d'autres diptères, et, en particulier, chez les Hippobosques, dont l'appareil reproducteur est très voisin de celui des Glossines.

L'anatomie générale de l'appareil reproducteur des femelles de Glossines est connue depuis le début du siècle (MINCHIN, 1905 ; STUHLMAN, 1907 ; ROUBAUD. 1909). Des observa- tions très détaillées sur la morphologie de l'appareil génital femelle et sur les transformations qu'il subit au cours de la gestation ont été effectuées par HOFFMANN (1954) chez Glossina palpolis. Mais ce n'est qu'en 1960 que SAUNDERS. en étudiant les ovaires de quałre espèces de glossines (Gl. morsitans. Gl. fuscipes fuscipes, Gl, pallidipes et Gl. brevipalpis) a établi la présence, dans chaque ovaire, de deux ovarioles, et a décrit le cycle de l'oogénèse des femelles de Gl. morsitans. VATTIER (1964) a étudié les caractères morphologiques et anatomiques des femelles capturées dans la nature, de Glossina palpalis palpolis et Glossina fuscipes quanzensis. CHALLIER (1965) a, d'après des études faites sur Glossina palpalis gambiensis. complété la méthode de diagnose de l'âge physiologique décrite par SAUNDERS.

Le présent travail apporte une contribution à la connaissance de l'age physiologique des. femelles de glossines en étudiant le cycle de l'oo- 


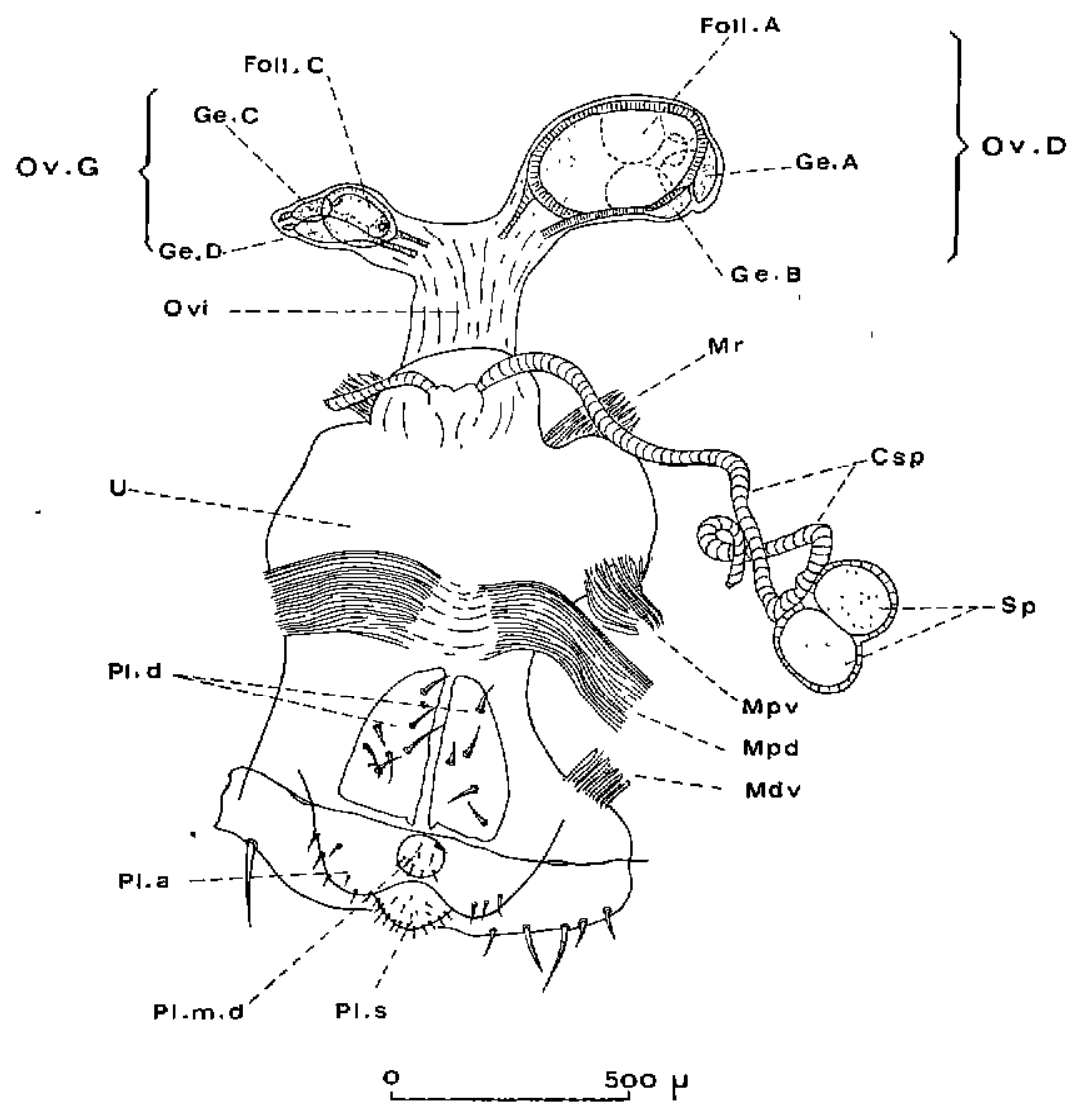

Fig. 1. - Organes génitaux d'une femelle de Gl. fachinoides âgée de 1 jour (la giande utérine a été enlevée).

\section{ABRÉVIATIONS}

Cellules nourricières $\ldots \ldots \ldots \ldots \ldots$ Cn

Chorion ...................... Ch

Conduit des spermathèques $\ldots \ldots \ldots$ Csp

Dent d'éclosion ................. De

Epithélium folliculaire............ Ep. foll.

Follicule .................... Foll.

Gaine de l'ovaire ................ GOv.

Gaine de l'ovariole ............... Go

Germarium ................. Ge

Glande utérine $\ldots \ldots \ldots \ldots \ldots$ Glu

Intima $\ldots \ldots \ldots \ldots \ldots \ldots \ldots$. In

Larve A2 ................... L. A2

Muscle dilatateur du vagin......... Mdv

Muscle protracteur dorsal .......... Mpd

Muscle protracteur ventral.......... Mpv

Muscle rétracteur ............... Mr

Noyaux des cellules nourricières.... NCn

Noyau de l'oocyte ................ NOo
Oeuf mûr.................... Om

Oocyte....................... Oo

Oviducte impair .................. Ovi

Ovaire droif ................. Ov. D.

Ovaire gauche $\ldots \ldots \ldots \ldots \ldots \ldots \ldots$ Ov. $G$.

Pédicelle....................... Ped

Plaque anale $\ldots \ldots \ldots \ldots \ldots \ldots$ PI. a.

Plaques dorsales ............... Pl. d.

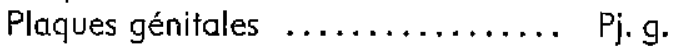

Plaques sternales .............. Pl.s.

Reliquat folliculaire ............ Rel, foll,

Restes de la gaine de l'ovariole ...... RGo

Saillies utérines................. Su

Sac ouvert $\ldots \ldots \ldots \ldots \ldots \ldots \ldots$. Sac

Spermathèques $\ldots \ldots \ldots \ldots \ldots \ldots \ldots$. Sp

Tube folliculaire $\ldots . \ldots \ldots \ldots \ldots$. Tfoll

Utérus ....................... U 
génèse chez des femelles de Glossina tachinoides West., élevées au laboratoire de l'Institut d'Elevage ef de Médecine vétérinaire des Pays tropicaux.

Dans un article précédent, nous avons décrit les méthodes d'élevage mises en œuyre au laboratoire, ainsi que les premiers résultats obtenus. Rappelons seulement que Glossina fachinoides a été élevée à partir de pupes importées du Tchad. Les imagos sont maintenus au laboratoire, à $25^{\circ} \mathrm{C}$ et $65-70$ p. 100 d'humidifé relative. Les pupes produites sont conservées à $25^{\circ} \mathrm{C}$ et $85 \mathrm{p}$. 100 d'humidité relative. Depuis quelques mois, toutefois. les imagos de Glossina tachinoides sont placés dans la même salle que les pupes $\left(25^{\circ} \mathrm{C}\right.$ ef 85 p. 100 d'humidité relative) et nourris chaque jour, sur lapin. Les accouplements ont lieu à l'âge de 3 jours pour les femelles et de 7 jours pour les mâles. Le pourcentage de fécondation est très satisfaisant et afteint près de 95 p. 100.

\section{I. - DESCRIPTION DE L'APPAREIL GÉNITAL DES FEMELLES DE GLOSSINA TACHINOIDES}

La morphologie de l'appareil reproducteur des femelles de glossines est en relation avec la larviparité. Cet appareil est formé de deux ovaires (un ovaire droit et un ovaire gauche) ; de deux oviductes pairs se réunissant pour constituer un oviducte impair, court, qui débouche dans la portion anférieure de l'utérus; de deux spermathèques avec leurs canaux et d'une glande nourricière, ou glande utérine (Fig. 1 et 2).

Chaque ovaire (Fig. 3) comprend deux ovarioles, un ovariole interne et un ovariole externe, couverts d'une membrane commune, la gaine ovarienne, richement pourvue de trachées, qui se prolonge du côté distal pour former les oviductes. Cefte membrane est constituée d'une couche de fibres musculaires. Chaque ovariole (deux par ovaire) est revêtu d'une membrane

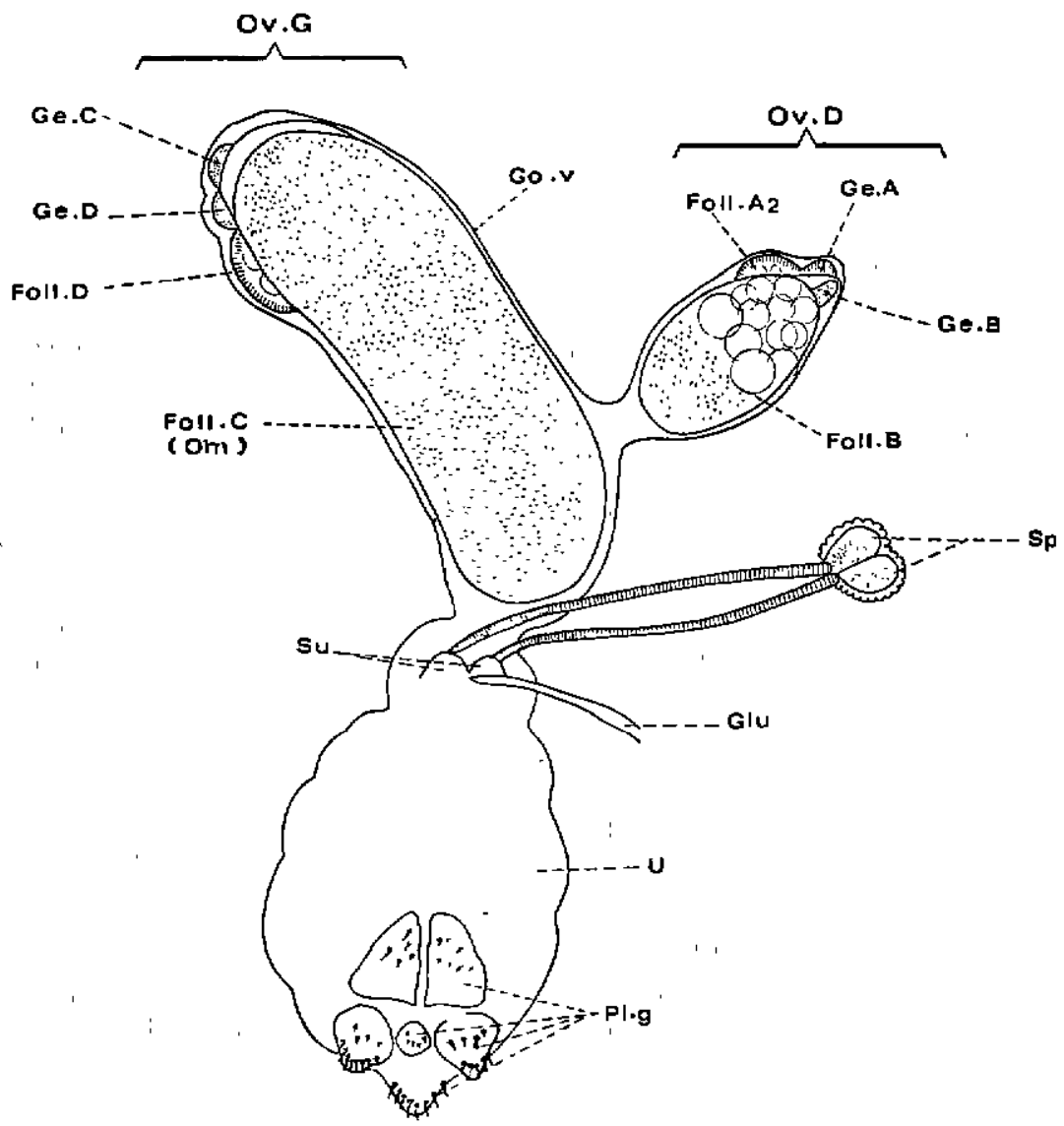

Fig. 2. - Organes génitaux d'une femelle de Gl. tachinoides âgée de 20 jours (1/2 schématique). 


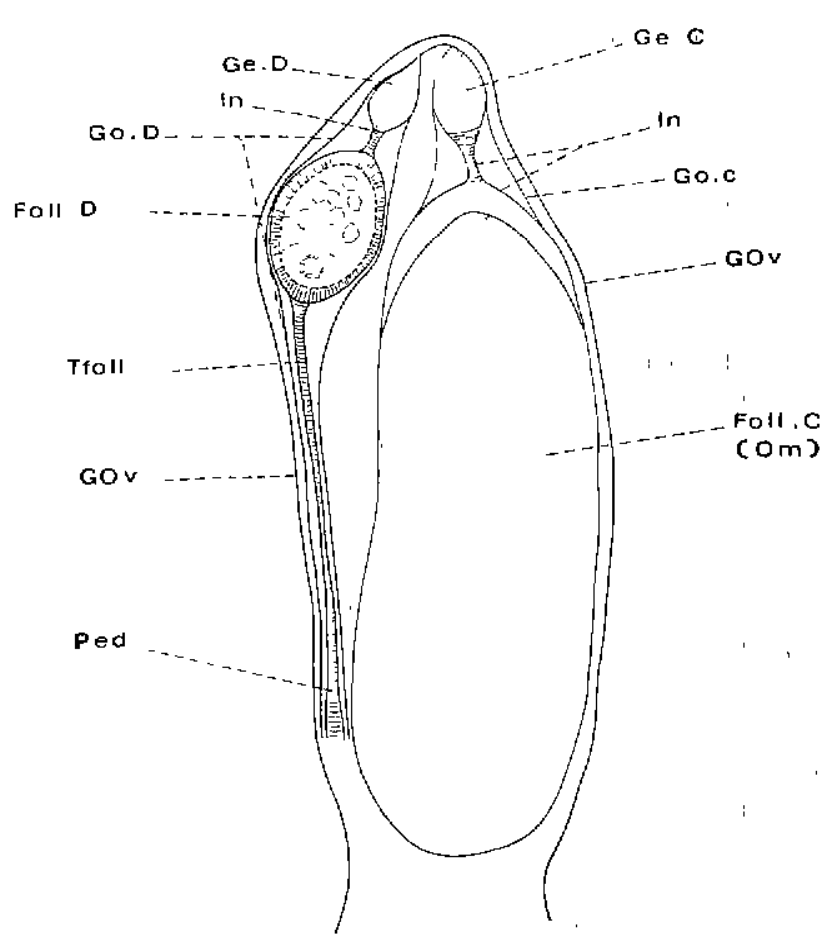

Fig. 3. - Ovaire gauche d'une femelle de Gl. tachinoides ôgée de 20 jours (schéma).

externe, ou gaine de l'ovariole, faite de tissu conjonctif làche et de fibres musculaires. Les deux gaines de l'ovariole, dans chaque ovaire, sont réunies, du côté proximal, par du tissu conjonctif. L'ovariole contient un follicule au-dessus duquel est situé le germarium ou chambre germinative, qui produit un nouveau follicule. L'ovariole tout entier est enveloppé d'une membrane, I'intima, adhérant étroitement au germarium ef au follicule, et doublant, à sa face interne, la gaine de l'ovariole. L'intima se prolonge, du côté distal, par un tube mince, le tube folliculaire, qui constitue la portion efférente de l'ovariole. L'extrémité postérieure du tube folliculaire, ou pédicelle, est quelquefois élargie et se fusionne à la paroi interne de la gaıne de l'ovariole.

L'oviducte impair débouche sur la partie dorsale de l'utérus, légèrement en arrière de son extrémité antérieure. Immédiatement après la jonction de l'oviducte avec l'utérus, la face dorsale de la paroi utérine présente, chez Glossıno tachinoides, deux sallies coniques accolées, à travers lesquelles pénètrent les conduits des spermathèques. Le canal ımpair de la glande utérine débouche légèrement en arrière de ces deux saillies (Fig. 2).

Les spermathèques, au nombre de deux, sont des réceptacles séminaux de forme globuleuse, étroitement accolés, de couleur brune. La face interne est formée d'une couche de chitine ornée de saillies. La face externe est entourée de cellules probablement glandulaires, fortement vacuolisées. communes aux deux spermathèques. De chaque spermathèque part un canal qui débouche au sommet de la papille dorsale correspondante de l'utérus. Ces canaux sont formés d'un revêtement cuticulaire spiralé recouvert d'une couche cellulaire et d'une couche musculaire à fibres longitudinales, qui se daublent de fibres circulaires vers la partie distale. pour former un sphincter.

La glande utérine est constituée d'un ensemble de tubes blanchâtres ramifiés, situés d̀ la face dorsale de l'utérus, et dont le canal, impair, aboutit à la base des saillıes dorsales utérines. Les dimensions et le nombre des ramifications varient suivant l'état de gestation. Chez les femelles gravides les ramifications sont plus nombreuses 
que chez les jeunes femelles. Ces glandes sont formées d'un épithélium simple recouvert d'une membrane. Le produit de sécrétion contient des globules graisseux et une masse granuleuse probablement de nature albuminoide. ll sert à nourrir la larve qui n'en utilise cépendant qu'une partie, le reste étant mis en réserve dans l'estomac et utilisé au moment de la pupaison.

L'utérus est une poche très extensıble située dans la partie ventrale de l'abdomen. Ses dimensions varient selon le stade de la gestation. En plein développement, il occupe la presque totalité de la cavité abdominale, refoulant vers la partie antérleure de l'abdomen les organes digestifs. A l'état de vacuité, il $a$, chez Glossina tachinoides, une forme grossièrement ovale, et présente généralement une série d'étranglements. Il est maintenu dans la cavité abdominale par différents muscles: muscles rétracteurs, antérieurs; muscles protrateurs, dorsaux et ventraux; muscles dilatateurs du vagın (Fig. 1). La paroi de l'utérus comprend une couche, externe, de muscles longitudinaux, circulaires ou obliques et une couche épithéliale, interne, garnie d'un mince revêtement chitineux.

Sur le plancher de l'utérus existe un épaississement longitudinal, le choriothète, formé d'un épithélium glandulaire et d'une partie musculaire. Cet organe aurait pour rôle d'agripper le chorion de l'ceuf, puis de le repousser après qu'il ait été percé par la dent orale de l'œuf. Le choriothète se fixe ensuite sur le tégument de la larve au 1er stade, puis dégénère lorsque celle-ci atteint le $2^{\mathrm{e}}$ stade. Au moment du dépôt de la larve, le choriothète se développe à nouveau ef atteint sa taille maximum lorsque l'œuf suivant descend dans l'utérus (BURSELL ef JACKSON, 1957). Un riche réseau trachéen, particulièrement développé sur la face ventrale et les faces latérales, entoure l'utérus. Un tissu adipeux, formé de cellules alignées en chainette, tapisse les faces externes de cet appareil qui débouche à l'extérieur par l'orifice génital situé entre les plaques génitales.

\section{II. - TECHNIQUE D'ÉTUDE}

L'étude du cycle de l'oogenèse a été effectuée sur les femelles de Glossina tochinordes élevées au laboratore, à $25^{\circ} \mathrm{C}$ et $70 \mathrm{p} .100$ d'humidité relative. Toutes les femelles mortes depuis moins de 2 heures d'âge connu, ont été disséquées dans une goutte de sérum physiologique, sous la loupe bınoculaire, ̀̀ l'aide de fines aiguilles montées. La dissection de l'appareil génital s'effectue de la façon suivante :

- Séparation de l'abdomen et du thorax à l'aide de fins ciseaux.

- Maintien en place, dans une goutte d'eau physiologique, de l'abdomen reposant sur la lame face inférieure vers le haut, à l'aide d'une aigulle montée tenue de la main gauche. Section du pourtour de l'abdomen, à l'exception du dernier segment, le long de la ligne de suture de la face supérieure et de la face inférieure, au moyen d'une aiguillle lancéolée finement aiguisée, tenve de la main droite.

- Dilacération des attaches du contenu abdominal aux pleures de la face supérieure de l'abdomen, puis section de cette face entre le dernier et l'avant dernier segment. Même opération avec la face inférieure de l'abdomen.

Une fois le contenu' abdominal dégagé, isolement, par dilacération ménagée, de l'appareil génital tout entıer, puis orientation de cet appareil en se basantsur la position des spermathèques.

Les dissections, effectuées très peu de temps après la mort, permeftent d'observer les mouvements péristaltiques du tube digestif et des déplacements, dans le sens antéropostérieur et postero-antérieur, des ovarioles à l'intérieur des gaines de l'ovaire. Ces mouvements sont très limités en amplitudes.

On peut également observer des mouvements brusques de forsion des conduits des spermathèques, cinsi que des mouvements péristaltiques des larves in ufero.

Cette méthode, quoique assez longue et délicate, offre l'avantage de ne pas léser les organes génitaux et de les conserver dans leurs positions respectives.

On note ensuite :

- La présence ou l'absence de spermatozoides dans les spermathèques, ef le volume qu'ils occupent.

- La présence ou l'absence d'œuf ou de larve dans l'utérus.

- La posifion, suivant leur taille respective, des ovarioles, dans chaque ovaire, et leur numé- 


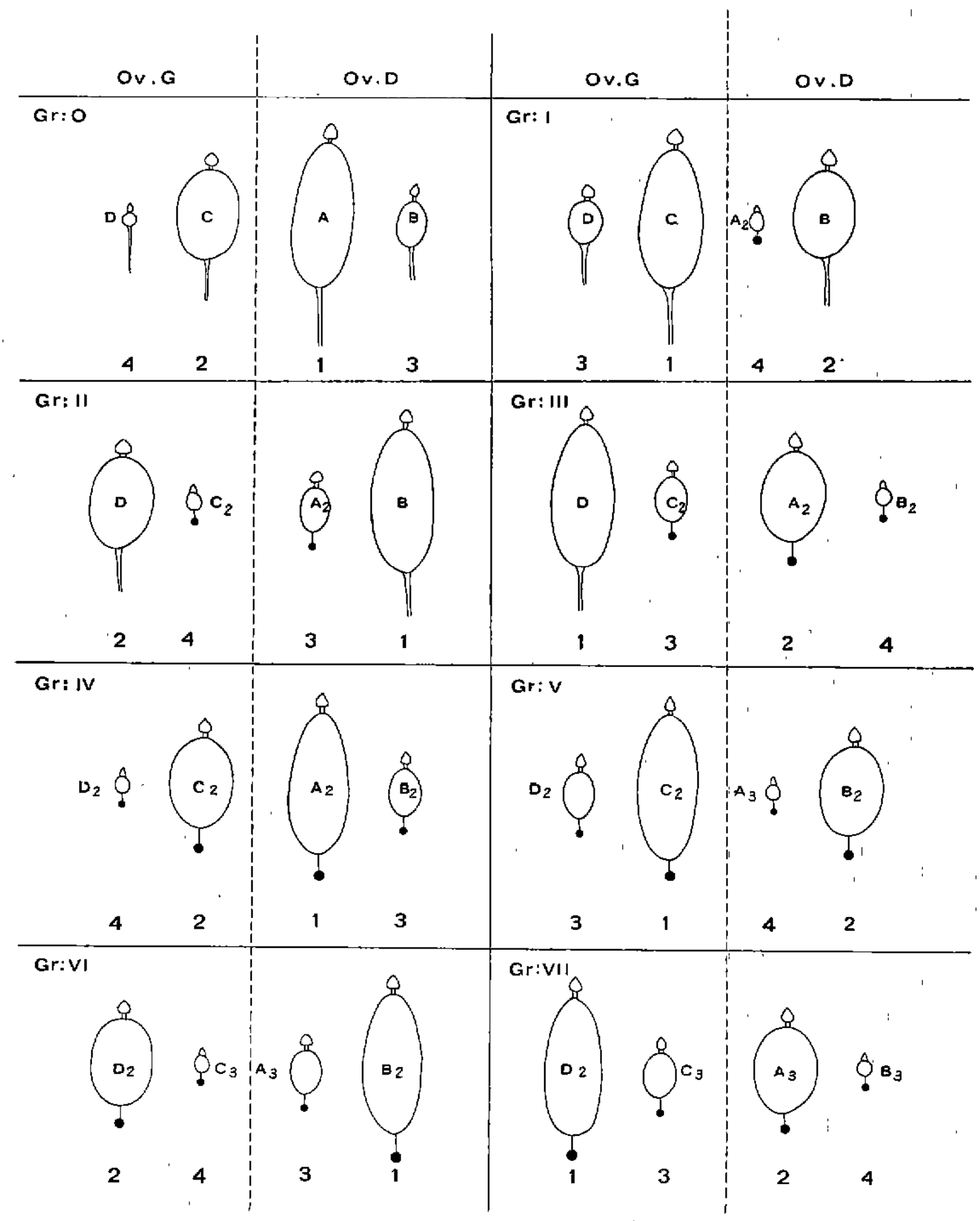

Fig. 4. - Tableau de diagnose de l'âge chez les femelles de Gl. tochinoides (le reliquat folliculaire est représenté par un point). 


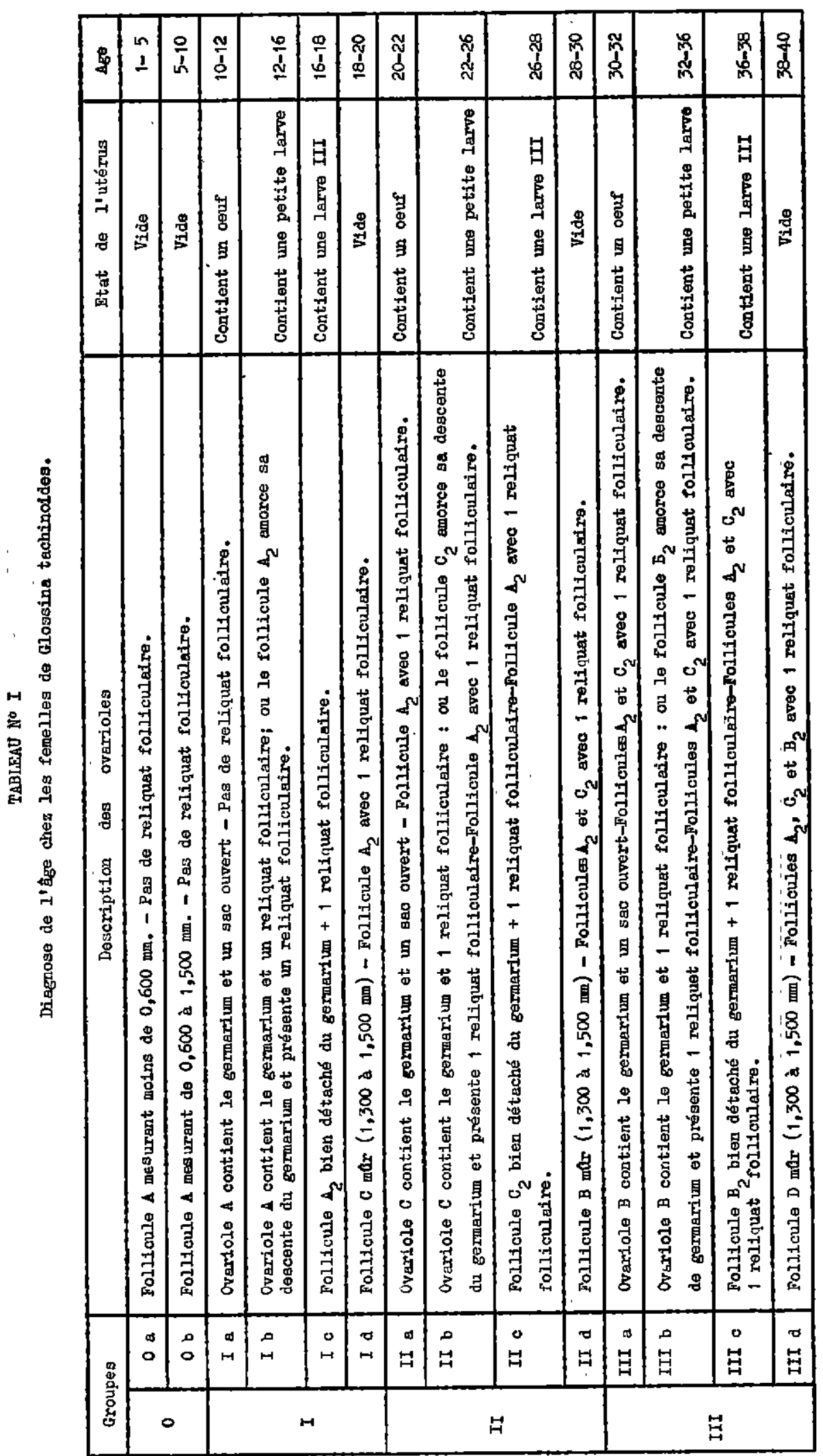




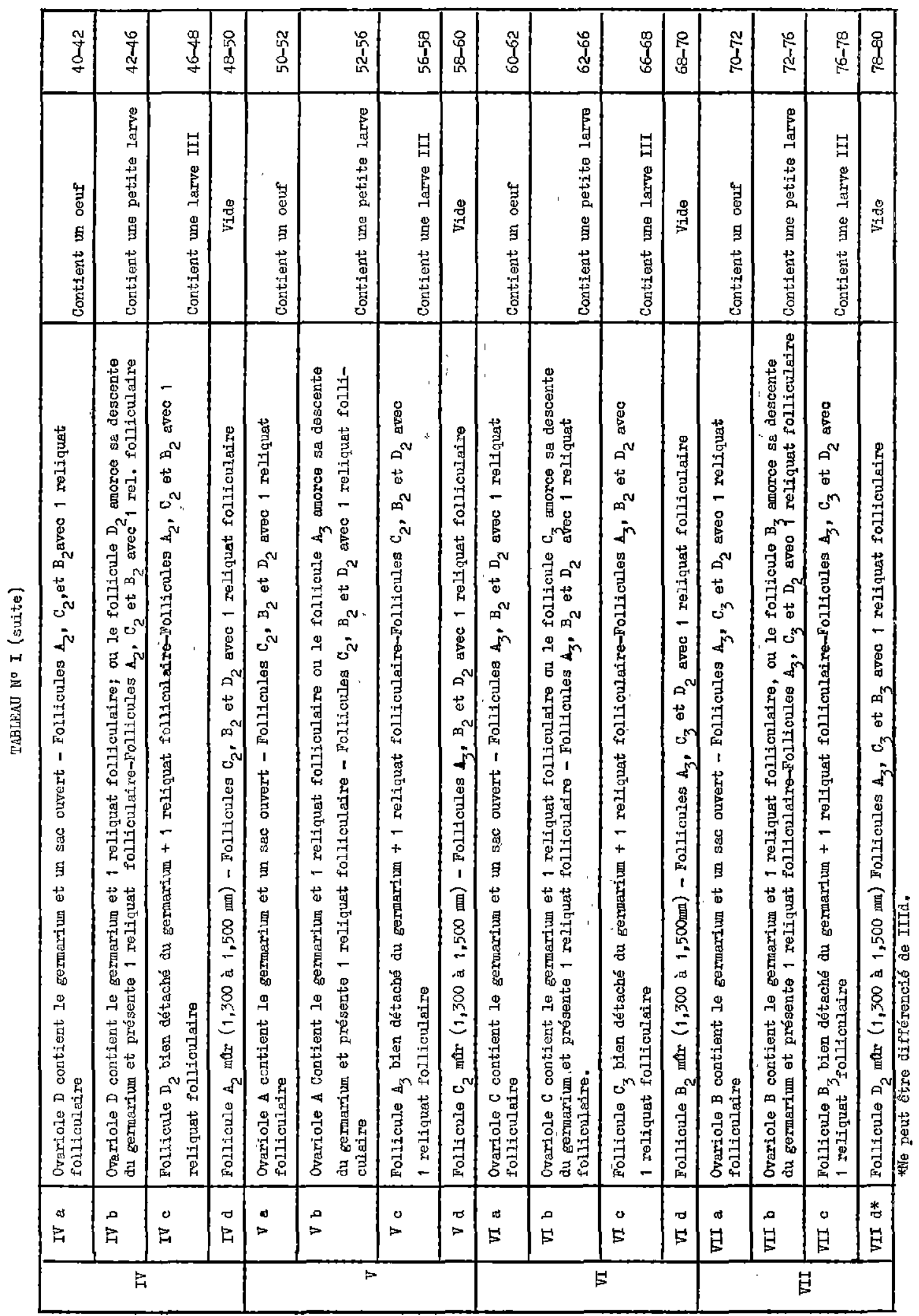


rotation selon la méthode décrite par CHALLIER (1964) (Fig. 4). Cette méthode consiste d̀ affecter à chaque follicule un numéro d'ordre correspondant d̀ sa taille, dans l'ordre de taille décroissante, sans tenir compte de leur position $(1,2,3$, 4), puis à assembler ces chiffres selon la position relative, dans l'espace, des ovarioles. Ainsi, le chiffre 4213 indique que le plus grand follicule se trouve dans l'ovariole interne de l'ovaire droit, et le plus petit dans l'ovariole externe de l'ovaire gauche.

Les ovaires sont ensuite séparés de l'utérus et chacun d'eux disséqué afin de dégager les ovarioles. On note alors, pour chaque ovariole, la présence d'un pédicelle ou d'une relique folliculaire, puis on procède à sa mensuration sous le microscope binoculaire. Les quatre ovarioles et l'œuf, ou la larve, s'il y en a une, sont montés en préparation définitive, au P. V. A. additionné d'une ou deux gouttes d'orcéine acétique au $1 / 100$.

Le nombre de follicule possédant une relique, leur position dans l'espace, leur dimension, l'absence, ou la présence, dans l'utérus, d'un œuf. d'une petite larve (larve au stade I ou II), ou d'une larve au stade III, permettent de déterminer le nombre de pupes produites par une femelle et de connaître son age physiologique.

\section{III. - OOGÉNÈSE NORMALE CHEZ GLOSSINA TACHINOIDES}

Chez les glossines, comme chez tous les diptères hématophages, les follicules sont du type polytrophique. L'ovariole contient à la fois les cellules nourricières ef l'oocyte (Fig. 5 ef 6 ). Du côté proximal se trouve le germarium. Le germarium est constitué d'épithélium germinatif et donne naissance aux cellules nourricières, et à d'autres cellules qui deviennent l'épithélium folliculaire. Chez Gl, tachinoides, le germarium donne naissance à 8 cellules filles (observations effectuées au microscope à contraste de phase). L'une de ces cellules deviendra l'oocyte et les sept autres les cellules nourricières. Ce groupe de cellules constitue un follicule. Ce n'est que dans les follicules récemment détachés du germarium que l'oocyte ne se différencie pas des cellules nourricières. Le jeune follicule se re- couvre d'une couche d'épithélium follıculaire et descend dans la lumière du tube folliculaire. Le follicule passe, au cours de son développement. par une série de stades que l'on peut décrire comme suit :

a) Le jeune follicule comprend 8 cellules indifférenciées. Il est sphérique et mesure, chez Gl. tachinoides, $0,090 \mathrm{~mm}$ environ.

b) Un oocyte, situé dans la partie distale du follicule, est nettement différencié et des globules de vifellus apparaissent autour de son noyau. Au-dessus de l'oocyte se trouvent les sept cellules nourricières. Le follicule, nettement détaché du germarium, commence à s'ovaliser ef mesure environ $0,160 \mathrm{~mm}$ d̀ $0,180 \mathrm{~mm}$ (Fig. 5).

c) Dans le protoplasme de l'oocyte, autour du noyau, les globules de vitellus sont plus gros et plus nombreux ef masquent le noyau. L'œuf croit, devient beaucoup plus gros que les cellules nourricières, qui se sont multipliées par divisions successives. L'œuf occupe plus de la moitié du follicule, qui prend une forme ovulaire (Fig. 6).

d) Le follicule s'allonge encore et le résidu des cellules nourricières n'en occupe que le sommet. L'oocyte, plein de vitellus et bien développé, occupe la totalité du follicule, qui, prêt à être ovulé, est mûr et mesure environ 1,5 mm. Le chorion couvre entièrement l'œuf.

II n'y a jamais, dans l'oogénèse normale, plus d'un follicule dans l'ovariole, le second follicule descendant du germarium plusieurs jours après l'ovulation du premier œuf.

Chez une femelle nouvellement éclose, les quatre ovarioles sont tous à des stades différents. Le plus grand follicule est dans l'ovariole interne de l'ovaire droit (il mesure, chez Gl. tachinoides, environ $0,450 \mathrm{~mm}$ ). Le follicule suivant le plus avancé est dans l'ovariole interne de l'ovaire gauche. Il mesure environ 0,150 à $0,200 \mathrm{~mm}$. L'ovariole externe de l'ovaire droit contient un follicule encore largement accolé au germarium, et l'ovariole externe de l'ovaire gauche ne contient que le germarium.

SAUNDERS (1960) désigne les ovarioles des femelles nouvellement écloses de la façon suivante:

Le plus gros ovariole de l'ovaire droit est désigné par la lettre A. 


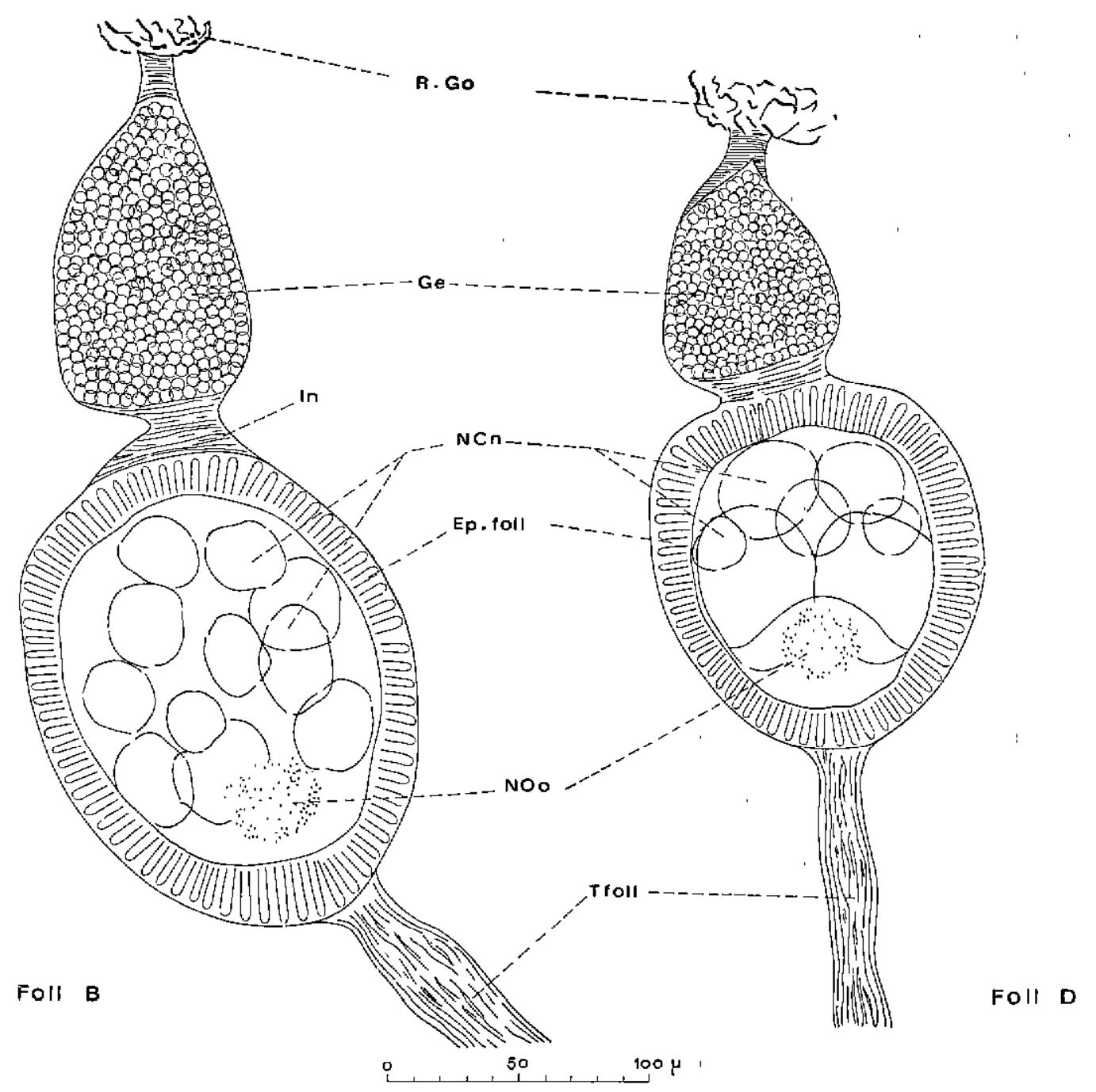

Fig. 5. - Ovariole externe de I'ovaire droit (B) et externe de l'ovaire gauche (D) d'une femelle de Gl. tachinojdes âgée de 10 jours'.

Le plus petit ovariole de l'ovaire droit est désigné par la lettre $B$.

Le plus gros ovariole de l'ovaire gauche est désigné par la lettre $C$.

Le plus petif ovariole de l'ovaire gauche est désigné par la lettre $D$.

CHALLIER (1964) a émis I'hypothèse, que nos dissections semblent confirmer, selon laquelle l'ovulation s'effectue dans un ordre constant :

1. Ovariole interne de l'ovaire droif (A).

2. Ovariole interne de l'ovaire gauche (C).

3. Ovariole externe de l'ovaire droit (B).

4. Ovariole externe de l'ovaire gauche (D).
Les ovarioles fonctionnant dans l'ordre A, C, $B, D$, les follicules correspondants sont appelés $A_{1}, C_{1}, B_{1}, D_{1}, A_{2}, C_{2}, B_{2}, D_{2}$, etc...

Au cours de l'ovulation, sous l'effet de la contraction des muscles ovariens, l'œuf est chassé vers l'extrémité distale de l'ovariole et traverse le tube folliculaire qui se distend considérablement. Après l'expulsion de l'cuf mûr, on trouve à l'endroit de son développement, en dessous du germarium, l'intima distendue, qui forme un sac mince, dont la longueur correspond à la longueur totale de l'œuf et du pédicelle. Ce sac contient, à son extrémité proximale, les restes des cellules nourricières et de l'épithélium folli- 


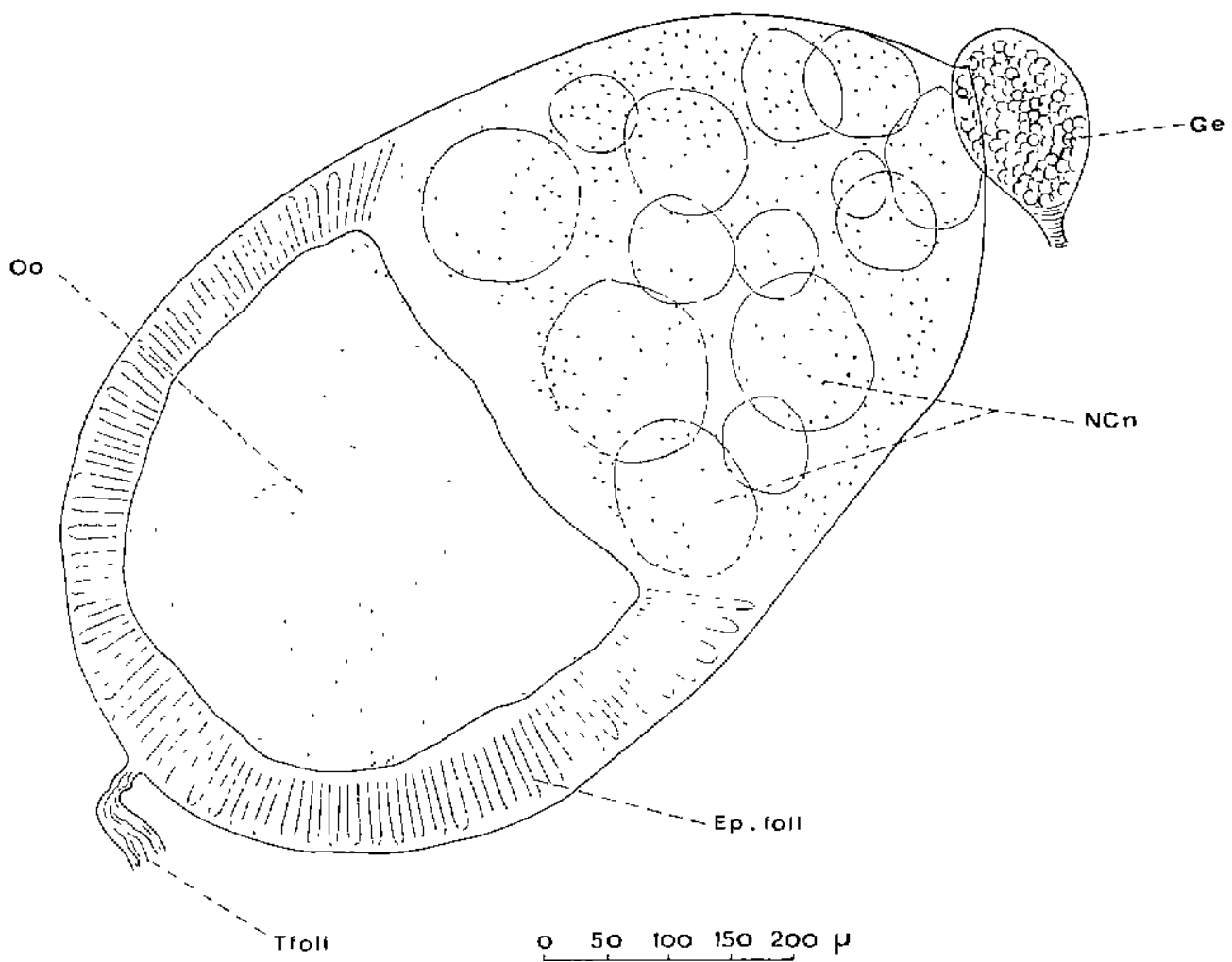

Fig. 6. - Ovariole interne de l'ovaire gauche (C) d'une femelle de Gl. tochinoides âgée de 10 jours.

culaire, qui forment une masse pigmentée jaunâtre.

Dans les jours suivant l'ovulation, le sac, qui était ouvert, se rétracte ef se débarrasse progressivement de la plus grande partie de ses inclusions. Lorsque l'intima est complèrement rétractée, le sac a été remplacé par une petife dilatation dans laquelle subsiste un petit nombre d'inclusions réfringentes. Lorsque l'œuf suivant se développera, cette dilatation formera à son extrémité distale un pefit appendice, que nous désignerons sous le terme de relique folliculaire (Fig. 7 ef 8 ).

Chez les glossines, lorsque le deuxième œuf d'un même ovariole est ovulé, la dilatation témoin de l'ovulation précédente est, dans la majorité des cas, expulsée avec l'ceuf. On ne trouve done le plus souvent, dans un ovariole, quelque soit le nombre d'ovulations qu'une seule relique folliculaire. Il est donc pratiquement impossible de reconnaître les ovarioles ayant ovulé deux fois ou plus, de ceux qui n'ont ovulé qu'une fois.

L'œuf, qui mesure entre 1,450 ef $1,570 \mathrm{~mm}$ chez Gl. tachinoides, possède un chorion dont la surface porte une réticulation polygonale très nette. L'extrémité postérieure de l'ceuf est plus large que l'extrémité antérieure, où se trouve le micropyle entouré du chorion transparent à cet endroit.

Chez Glossina fachinoides, à $25^{\circ} \mathrm{C}$ et $70 \mathrm{p.} 100$ d'humidité relative, la première ovulation se produit vers le $9 \mathrm{e}_{-10}$ jour, quelquefois plus tard (vers le $11^{\circ}$ ou $12^{\mathrm{e}}$ jour). L'œuf provenant de l'ovariole $A$, est fécondé lorsque le micropyle arrive au niveau de l'orifice du conduit des spermathèques. 


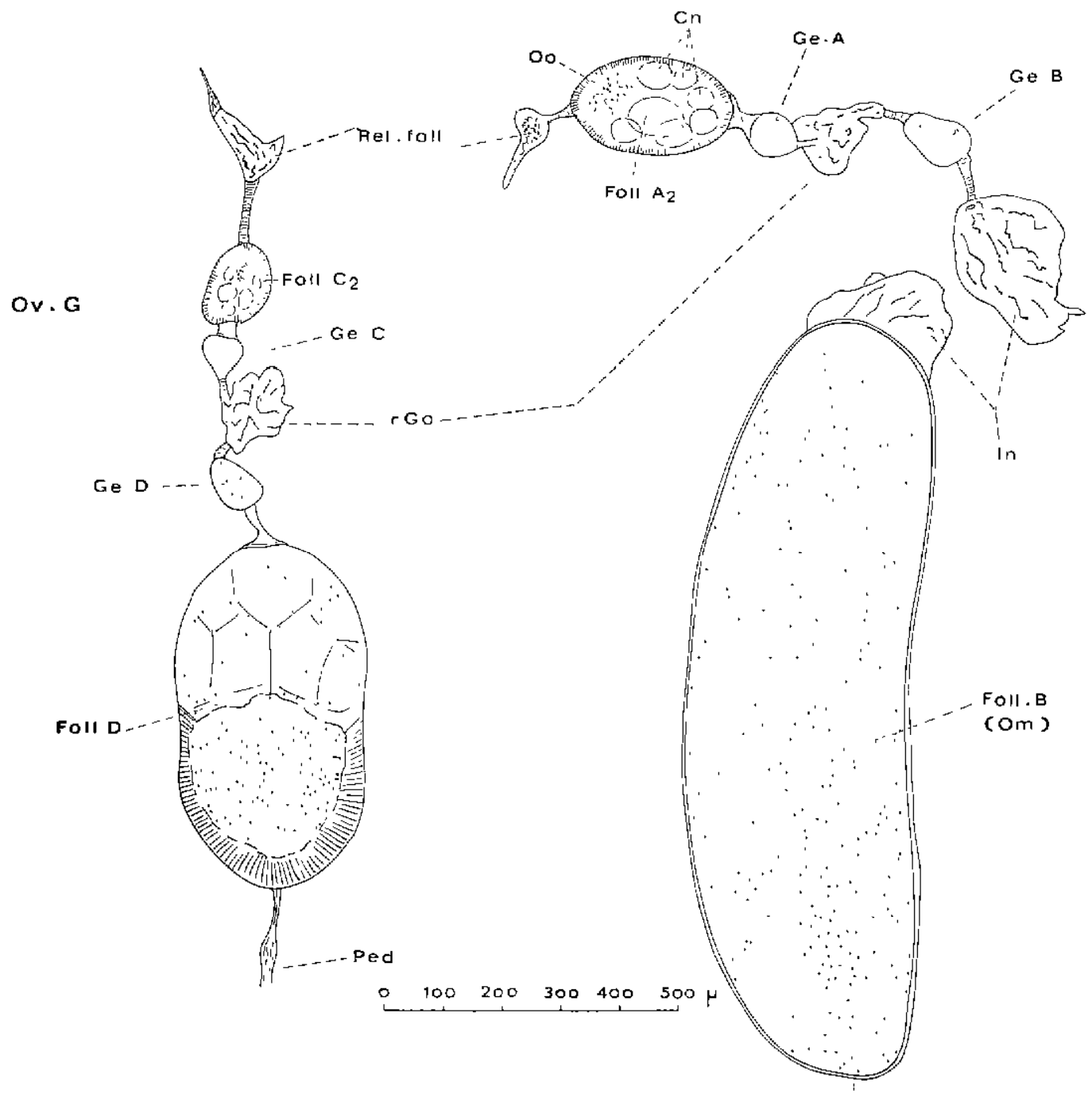

OV.D

Fig. 7.-Les quatre ovárioles d'une femelle de Gl. fachinoides âgée de 30 jours (l'intima du follicule $B$ a été déchirée en cours de dissection).

La larve atteint le stade $f l i$ en 6 jours environ. La première ponte a lieu entre le $17 \mathrm{e}$ et le $20 \mathrm{e}$ jour, parfois un peu plus tard. L'œuf suivant, produit par l'ovariole $C$, se trouve dans l'utérus vers le $20-22$ e jour. Le follicule $A_{2}$ présente à ce moment une relique folliculaire, témoin de la première ovulation. La deuxième larve est pondue vers le $26 e_{-} 30$ e jour. Les pontes successives ont lieu, après la première, tous les 810 jours, en moyenne.

$L$ 'œuf provenant de l'ovariole $B$ est ovulé vers le $30 \mathrm{e}-32^{\mathrm{e}}$ jour, et l'œuf provenant de l'ovariole
$D$, vers le $40^{\ominus}-42^{e}$ jour. Le deuxième œuf produit par l'ovariole $A$ (follicule $A_{2}$ ) sera ovulé vers le $50^{\circ}-52^{\circ}$ jour.

Dans la plupart des cas les œufs ne succèdent pas immédiatement, dans l'utérus, à la ponte de la larve précédente. II s'écoule généralement un intervalle d'un à deux jours entre la ponte d'une larve au dernier stade ef l'ovulation suivante. II est done fréquent, chez une femelle ayant, d'après le nombre de reliques folliculaires présentes dans les ovarioles, produit une ou plusieurs pupes, de trouver un utérus vide. 

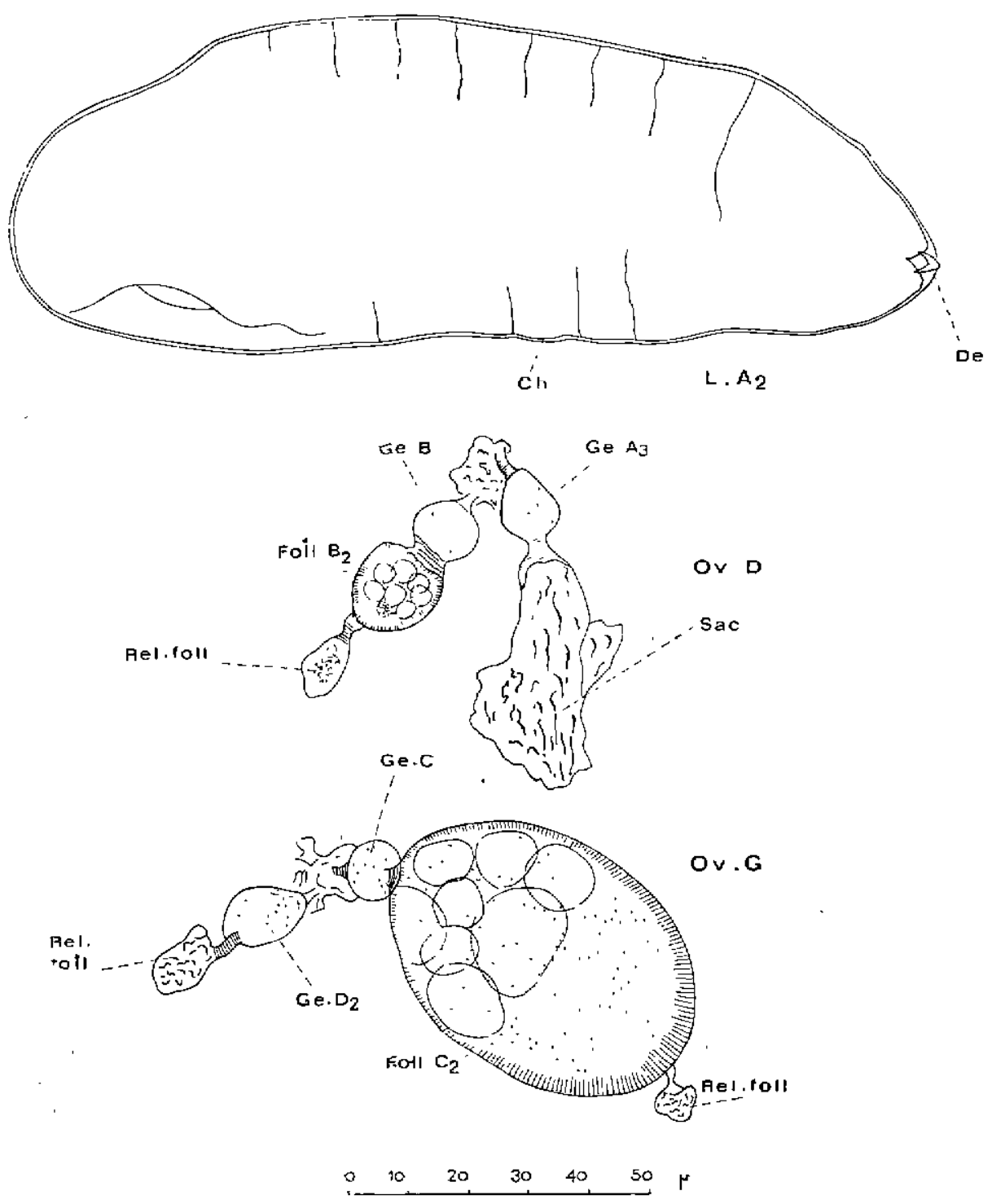

Fig. 8. - Les quatre ovarioles d'une femelle de $G$. tachinoides âgée de 50 jours.

L'utérus contenant un ceuf provenant du follicule $A_{2}$.

\section{IV. - DIMENSION DES FOLLICULES CHEZ LES FEMELLES DE GLOSSINA TACHINOIDES}

Nous avons, lors de la dissection des ovarioles, mesuré au microscope binoculaire la plus grande dimension de chaque follicule, à l'exclusion du germarium et du tube folliculaire. Les mensurations ont été effectuées au micromètre oculaire, le coefficient micrométrique étant de 10 avec l'objectif $\times 10$ et de 5 avec l'objectif $\times 20$.
Il n'a pas été possible d'effectuer une étude statistique, en fonction de l'âge, des mensurations relevées, le nombre de femelles disséquées dans chaque groupe d'âge n'étant pas suffisant.

Les dimensions que nous indiquons ci-après et dont le tableau II rassemble quelques exemples ne constituent donc qu'un ordre de grandeur.

Chez la femelle nouvellement éclose ou âgée de 1 jour, le follicule $A$ mesure $0,455 \mathrm{~mm}$ ef le follicule $C$ mesure $0.160 \mathrm{~mm}$. Le follicule $B$ n'est pas encore détaché du germarium. 


\begin{tabular}{|c|c|c|c|c|c|}
\hline Age en jours & Ovariole $A$ & Ovariole B & Ovariole C & Ovariole D & Etat de l'utérus \\
\hline $\begin{array}{ll}1 \\
2 \\
3 \\
4 \\
5 \\
6 \\
7 \\
8 \\
9-10 \\
9-16 \\
15-18 \\
20 \\
20 \\
22 \\
25 \\
28 \\
32 \\
39 \\
56 \quad(1) \\
59 \quad(2) \\
60 \quad(3)\end{array}$ & $\begin{array}{c}0,455 \\
0,480 \\
0,510 \\
0,530 \\
0,515 \\
0,756 \\
1,250 \\
1,375 \\
1,500 \\
\text { germ. + rel. } \\
0,100+\text { rel. } \\
0,113+\text { rel. } \\
0,163+\text { rel. } \\
0,163+\text { rel. } \\
0,150+\text { rel. } \\
0,260+\text { rel. } \\
0,263+\text { rel. } \\
0,465+\text { rel. } \\
0,412+\text { rel. } \\
0,065+\text { rel. } \\
0,188+\text { rel. }\end{array}$ & $\begin{array}{c}\text { a.f. } \\
0,088 \\
0,145 \\
0,115 \\
0,100 \\
0,110 \\
0,163 \\
0,150 \\
0,188 \\
0,238 \\
0,413 \\
0,438 \\
0,525 \\
1,233 \\
1,450 \\
1,325 \\
\text { germ. + sac } \\
0,135+\text { rel. } \\
0,112+\text { rel. } \\
0,400+\text { rel. } \\
1,475\end{array}$ & $\begin{array}{c}0,160 \\
0,175 \\
0,190 \\
0,198 \\
0,206 \\
0,221 \\
0,313 \\
0,388 \\
0,825 \\
1,025 \\
1,438 \\
1,500 \\
\text { germ. + sac } \\
\text { germ. + sac } \\
\text { germ. + rel. } \\
0,125+\text { rel. } \\
0,150+\text { rel. } \\
0,200+\text { rel. } \\
0,162+\text { rel. } \\
1,563 \\
0,125+\text { rel. }\end{array}$ & $\begin{array}{l}\text { germ. } \\
\text { germ. } \\
\text { germ. } \\
\text { germ. } \\
\text { germ. } \\
\text { d.f. } \\
0,088 \\
0,113 \\
0,175 \\
0,163 \\
0,200 \\
0,187 \\
0,225 \\
0,250 \\
0,300 \\
0,485 \\
1,313 \\
1,570 \\
\text { germ.trel. } \\
0,213+\text { rel } \\
0,438+\text { rel }\end{array}$ & $\begin{array}{l}\text { Vide } \\
\text { Vide } \\
\text { Vide } \\
\text { Vide } \\
\text { Vide } \\
\text { Vide } \\
\text { Vide } \\
\text { Vide } \\
\text { Vide } \\
\text { Larve I } \\
\text { Vide } \\
\text { Vide } \\
\text { Larve I } \\
\text { Larve II } \\
\text { Larve II } \\
\text { Vide } \\
\text { Larve I } \\
\text { Vide } \\
\text { Larve I } \\
\text { LarveIII } \\
\text { (lobes noirs) } \\
\text { Vide }\end{array}$ \\
\hline
\end{tabular}

(1) - 1ère larve pondue le 36ème jour ; 2ème larve pondue le 44 ème jour ; 3ème larve pondue le 54 ène jour.

(2) - tère larve pondue le 19 ème jour ; 2ème larve pondue le 27ème jour ;

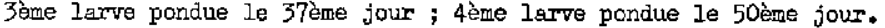

(3) - lère larve pondue le 17ère jour ; Lème larve pondue le 25 ème jour; 3ème larvo pondue le 35 ème jour ; 4ème larre pcndue le 45 ème jour; 5ème larve pondue le 52ème jour ; 6ème larve pondue le 58ème jour.

Tablequ II. - Variations de la longueur des follicules en fonction de l'âge chez Gl. tochinoides.

(Germ., germarium ; rel., présence d'un reliquat folliculaire ; sac, présence d'un sac ouvert ; d. F. premier signe de la descente du follicule).

SAUNDERS indique. pour Gl. mortisans âgée de 0-1 jour les dimensions suivantes:

Follicule $\mathrm{A}=0,486 \mathrm{~mm}$; follicule $\mathrm{C}=$ $0,226 \mathrm{~mm}$.

Les dimensions du follicule A sont voisines dans les deux espèces ; par contre le follicule $C$ est nettement plus petit chez $G$. tachinoides.

Ces différences s'accentuent avec l'âge, puisque chez $\mathrm{Gl}$. tachinoides âgée de 8 jours, nous avons relevé les dimensions suivantes:

$$
\begin{aligned}
& A=1,375 \mathrm{~mm} . \\
& B=0,150 \mathrm{~mm} . \\
& C=0,388 \mathrm{~mm} . \\
& D=0,113 \mathrm{~mm} .
\end{aligned}
$$

Chez Gl. morsitons àgée de 8 jours, SAUNDERS indique $1,600 \mathrm{~mm}$ pour $A ; 0,200 \mathrm{~mm}$ pour $B$; $0,506 \mathrm{~mm}$ pour $C$ et $0,165 \mathrm{~mm}$ pour $D$.

A l'âge de 20 jours, les dimensions sont, respectivement, chez Gl. tachinoides et Gl. morsitans :

$$
\begin{aligned}
& A=0,113 \mathrm{~mm} \text { et } 0,153 \mathrm{~mm} . \\
& B=0,438 \mathrm{~mm} \text { et } 0,463 \mathrm{~mm} . \\
& C=1,500 \mathrm{~mm} \text { et } 1,600 \mathrm{~mm} . \\
& D=0,187 \mathrm{~mm} \text { et } 0,250 \mathrm{~mm} .
\end{aligned}
$$

\section{V. - DÉVELOPPEMENT LARVAIRE}

L'œuf se place dans l'utérus avec l'extrémité antérieure dirigée vers l'avant. II est enveloppé du chorion et contient la larve'au premier stade, dont la structure interne est difficilement discernable. Cette larve possède une structure médiane chitinisée, la dent d'éclosion (Fig. 8) qui lui sert à déchirer le chorion, lequel se fend longitudinalement, sur la surface dorsale. Le chorion est repoussé par le choriothète et expulsé. Le premier stade larvaire ne durerait que quelques heures (ROUBAUD 1909 ; BURTT ef JACKSON 1951). Lorsque le chorion est expulsé, on peut distinguer les principaux troncs trachéens. La 


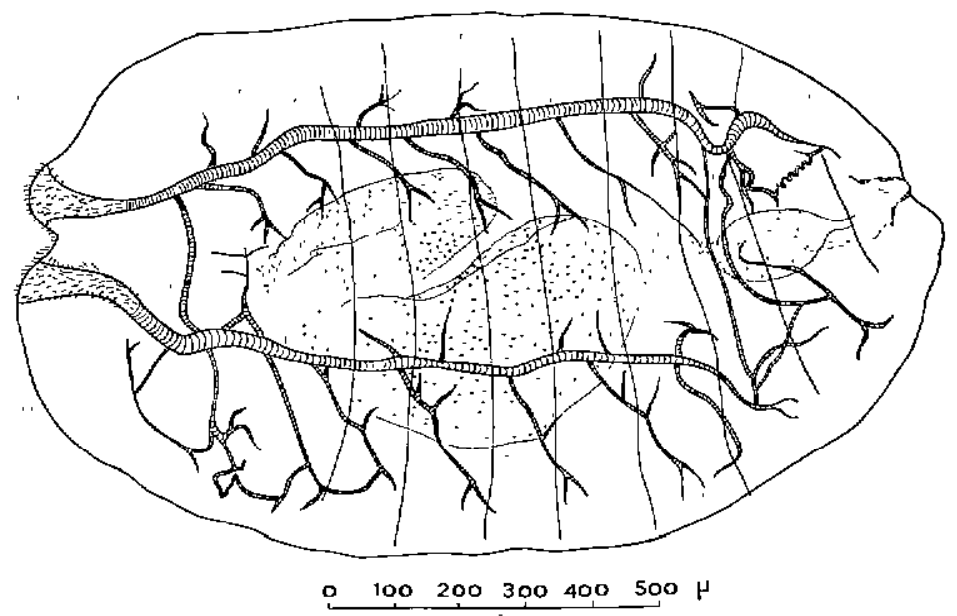

Fig. 9. - Gl. tachinoides, larve au 1er stade.

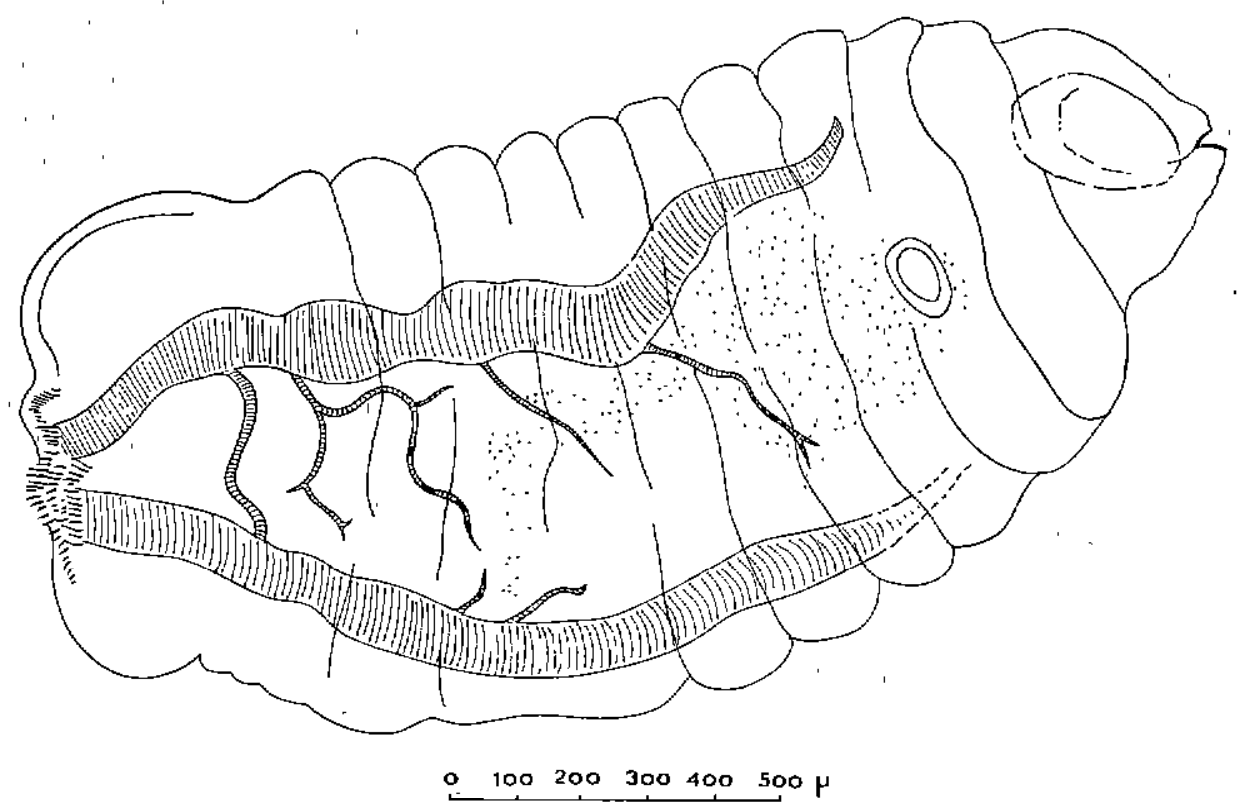

Fig. 10. - Gl. tachinoides, larve au $2^{\mathrm{e}}$ stade.

larve de premier stade mesure environ $1,6 \mathrm{~mm}$ (Fig. 9).

Chez la larve de deuxième stade (Fig. 10), les lobes respiratoires sont partiellement développés. Le tégument est recouvert d'épines. Durant ce stade la larve croît pour atteindre $3,5 \mathrm{~mm}$ environ à la deuxième mue.
Au troisième stade les lobes polypneustiques sont entièrement développés. Vers la fin de la vie utérine, ces lobes deviennent noirs ef sont visibles à travers le tégument de la mère. La masse antenno-maxillaire est parfaitement visible. La larve au troisième stade mesure environ, labes compris, $5,7 \mathrm{~mm}$ de longueur. 


\section{VI. - DÉTERMINATION \\ DE L'AGE PHYSIOLOGIQUE CHEZ LES FEMELLES DE GLOSSINA TACHINOIDES}

SAUNDERS $(1960,1962)$ a établi une méthode de diagnose de l'âge physiologique chez les femelles de Glossina morsitans basée sur l'absence ou la présence de reliques folliculaires. SAUNDERS classe les femelles de glossines en cinq groupes suivant que les différents ovarioles présentent ou non des dilatations avec relique folliculaire, L'absence de relique folliculaire permet de distinguer les femelles nullipares des femelles pares. Les femelles pares sont eiles-mêmes classées en groupes d'âge croissant suivant qu'elles présentent 1, 2, 3 ou 4 dilatations dans l'ensemble des ovarioles. C'est ainsi qu'appartiennent à :

- La catégorie 0 , les femelles nullipares, qui ne présentent aucune dilatation sur aucun des tubes folliculaires.

- La catégorie I, les femelles présentant une relique folliculaire dans le tube folliculaire de l'ovariole A.

- La catégorie II, les femelles présentant deux dilatations, une dans l'ovariole $\mathrm{A}$, une dans l'ovariole $C$.

- La catégorie III, les femelles présentant trois dilatations, une dans I'ovariole $A$, une dans l'ovariole $C$ et une dans l'ovariole $B$.

- La catégorie IV, les femelles présentant quatre dilatations, une dans l'ovariole $A$, une dans l'ovariole $C$, une dans l'ovariole $B$ et une dans I'ovariole D.

Dans chacune des catégories I, II, III et IV, SAUNDERS distingue en outre des groupes d'âge $a, b, c$, suivant que l'utérus contient un œuf, une petite larve (larve au stade I ou II) ou une larve au III stade.

Dans la catégorie IV sont incluses toutes les femelles ayant effectué plus de quatre ovulations. Les dilatations de chaque ovariole étant éliminées avec l'expulsion de l'œuf suivant, il n'est en effet pas possible, avec cette méthode, de distinguer les femelles ayant effectué 5,6 ovulations, ou plus, de celles, qui n'ont pondu que quatre fois.

CHALLIER (1964) a rendu cette méthode plus précise en formulant l'hypothèse selon laquelle les ovulations s'effecivent dans un ordre consiant : le premier œuf provient de l'ovariole A; le deuxième de l'ovariole $C$; le troisième de l'ovariole $B$; le quatrième de l'ovariole $D$; le cinquième à nouveau de l'ovariole $A$; le sixième de l'ovariole $C_{\text {, et ainsi de suite. En attribuant }}$ dans les ovaires intacts et en position normale, un numéro d'ordre par taille décroissante à chaque ovariole, et en groupant ces chiffres selon la position, dans l'espace, des ovarioles, on obtient un nombre repère caractéristique, qui, joint à l'étude du nombre de reliques folliculaires, permet de déterminer l'age d'une femelle jusqu'à la septième ovulation incluse. On peut ainsi distinguer huit groupes d'âge (tableau let Fig. 4):

$$
0-|-||-|||-|V-V-V|-V||
$$

Les groupes 0 , I, II ef III correspondent aux catégories 0,1 , II et III de SAUNDERS.

Le groupe IV comprend les femelles ayant effectué $(4+4 n)$ ovulations. Le groupe $V$ comprend les femelles ayant effectué $(5+4 n)$ ovulations. Le groupe $\mathrm{Vl}$ comprend les femelles ayant effectué $(6+4 n)$ ovulations et le groupe VIII les femelles ayant effectué $(7+4 n)$ ovulations.

Les dissections que nous avons faites sur les femelles de Glossina tachinoides provenant de notre élevage semblent confirmer la valeur de cette méthode. Les dimensions ef la position des ovarioles peuvent être aisément appréciées avant la dissection des ovaires car les follicules sont visibles à travers la gaine de l'ovaire. Les seuls nombres repères possibles sont (Fig. 4) :

4213, qui correspond à des femelles appartenant aux groupes 0 ou IV.

3142, correspondant à des femelles des groupes I ou $\mathrm{V}$.

2431, correspondant à des femelles des groupes II ou Vl.

1324, correspondant à des femelles des groupes III ou VII.

Il peut arriver que l'on obtienne un nombre repère théoriquement impossible. tel que 1342 . Cette anomalie se produit quelquefois lorsque I'un des ovarioles contient un ceuf mûr. II est probable que l'œuf mûr, en créant une tension sur la paroi de l'ovaire, a fait glisser le petit ovariole sur sa face concave. Il suffit alors d'intervertir les chiffres correspondant respectivement. dans le même ovaire; à l'œúf mûr et au petit ovariole. Dans l'exemple cité ci-dessus, le nombre repère réel est 3142 . 
Une imprécision subsiste cependant lorsqu'on ne peut être certain de la présence d'une relique folliculaire en dessous d'un œuf mûr dans l'ovariole externe gauche (ovariole D). L'œuf môr distend en effet considérablement le tube folliculaire, qui devient extrêmement fragile et se déchire lors de la dissection. Dans ces conditions, la dilatation-témoin de l'ovulation précédente se confond avec l'intima déchirée et n'est généralement pas repérable. Le groupe d'âge exact reste alors imprécis, la femelle pouvant appartenir aux.groupes III, VII, XI, etc...
Une autre imprécision provient de ce que, audelà du groupe VII, il n'est pas possible de caractérisẹr les femelles ayant eu 8 ovulations ou plus. Toutefois' cetfe imprécision n'affecie que les femelles âgées de plus de 80 jours. Or la répartition des effectifs par groupe d'âge diminue très rapidement à partir du groupe VII. Au laboratoire. la moyenne de vie des femelles de Gl. tachinordes a été, dans les meilleures canditions, de 50 jours, avec un maximum de 111 jours. Le nombre de femelles vivantes pour 100 femelles écloses, se répartit de la façon suivante : (Tableau III).

TABLEAT NOIII

\begin{tabular}{|c|c|c|c|c|c|c|c|c|c|c|c|c|}
\hline Age en jour & 0 & $1-10$ & $11-20$ & $21-30$ & $31-40$ & $41-50$ & $51-60$ & $61-70$ & $71-80$ & $81-90$ & $91-100$ & $101-\$ 10$ \\
\hline Groupe d'âge & 0 & 0 & 1 & II & III & IV & $\mathrm{V}$ & VI & VII & VIII & $I X$ & $x$ \\
\hline $\begin{array}{l}\text { Nombre de } \\
\text { fomelles } \\
\text { vivantes }\end{array}$ & 100 & 71 & 71 & 71 & 61 & 55 & 42 & 39 & 32 & 3 & 3 & 1 \\
\hline
\end{tabular}

CHALLIER, avec des femelles de Glossina palpalis gambiensis capturées en Haute-Volta, obtient la répartition par groupe d'áge suivante : (Tableau IV).
Cette méthode doit donc permettre de calculer, avec une précision suffisante, l'âge physiologique des femelles capturées dans la nature. La correspondance des groupes d'âge avec l'âge

TABLEAU NOIV

\begin{tabular}{|l|c|c|c|c|c|c|c|c|c|}
\hline Groupe dîge & 0 & I & II & III & IV & I,V & VI & VII & Total \\
\hline Effectif & 225 & 32 & 52 & 40 & 43 & 33 & 13 & 4 & 442 \\
\hline Pourcentage & 50,9 & 7,2 & 11,8 & 9,0 & 9,7 & 7,5 & 2,9 & 0,9 & 99,9 \\
\hline
\end{tabular}

réel a été calculée, pour Glossina fachinoides à partir de femelles d'âge connu élevées au laboratoire. Le tableau if indique cette correspondance. En fait, la concordance n'est pas rigoureusement exacte. Les chiffres indiqués ne correspondent qu'à des moyennes. Les conditions artificielles de l'élevage en laboratoire affectent dans une certaine mesure la physiologie des femelles. C'est ainsi que nous avons noté, dans un cas extrême, un retard dans la ponte de la première larve atteignant 16 jours (dépôt de la première larve au 36e jour suivant l'éclosion de la femelle). Le rythme de ponte se trouve décalé d'autant.

En outre, les pontes successives n'ont pas lieu à des intervalles rigoureusement constants. Nous avons ainsi noté des périodes interlarvaires minima de 6 jours et maxima de 13 jours, la moyenne se situant cependant aux environs du 9 e, $10^{e}$ jour.

\section{VII. - CONCLUSIONS}

Glossına tachinoides West., espèce du sousgenre Nemorhino (= groupe palpalis), a une biologie très particuliere qui la rapproche de certaineś espèces du sous-genre Glossino (= groupe morsitans). Cette particularité se traduit dans la nature par une répartition géographique originale. Des élevages de Gl. tochinoides n'ont été que très rarement réalisés (BUXTON et LEWIS, 
1934. GRUVEL et BALIS, 1965). De ce fait, cette espèce n'a été que peu étudiée.

Ayant pu obtenir, pendant plus d'un an, au laboratoire, un élevage de Gl. tachinoides à partir de pupes importées du Tchad, il nous a été possible d'étudier pour la première fois le cycle de l'oogénèse chez cette espèce.

L'oogénèse, chez les femelles de Gl. tochinoides West, élevées au laboratoire, à $25^{\circ} \mathrm{C}$ et $70 \mathrm{p}$. 100 d'humidité relative, et fécondées à l'âge de 3 jours, suit un cycle sensiblement identique à celui déjà décrił par différents auteurs chez Gl. morsitans, Gl. fuscipes fuscipes, $G l$. pallidipes et Gl. brevipalpis (SAUNDERS, 1960, 1961, 1962) ; chez Gl. palpalis palpalis et Gl. fuscipes quanzensis (VATTIER, 1964) ; chez Gl. palpalis gombiensis (CHALLIER, 1964 et 1965).

Chez la femelle nouvellement éclose, les quatre ovarioles sont tous à des stades différents. Le follıcule le plus développé se trouve dans l'ovariole interne de l'ovaire droit, ef le moins développé dans l'ovariole externe de l'ovaire gauche.

Le jeune follicule comprend 8 cellules, dont une deviendra l'oocyte et les 7 autres formeront les cellules nourricières.

La première ovulation a lieu vers le $9 \mathrm{e}$ jour suivant l'éciosion de la femelle, et la première larve est déposée vers le $18 \mathrm{e}$ jour. Les pontes suivantes ont lieu à des intervalles de 9 à 10 jours.

Des retards dans l'ovulation ou le rythme de ponte ont été constatés chez quelques femelles. Ces anomalies sont vraisemblablement imputables aux conditions artificielles d'élevage en laboratoire.

Les dimensions des follicules, chez Gl. tachinoides, sont sensiblement inférieures à celles indiquées par SAUNDERS chez Gl, morsitans.
L'œuf mûr, en particulier, mesure environ $1,5 \mathrm{~mm}$ chez Gl. tachinoides, contre $1,6 \mathrm{~mm}$ chez $\mathrm{Gl}$. morsitans.

If he subsiste dans la grande majorité des cas, après l'ovulation, qu'une seule dilatation dans le tube follicullaire. II n'y a pas, comme chez les moustiques, de séries de dilatations ef il n'est donc pas possible de distinguer les ovarioles ayant ovulé deux fois ou plus de ceux qui n'ont ovulé qu'une fois. Néanmoins, en se basant sur I'hypothèse formulée par CHALLIER, hypothèse que les dissections effectuées chez Gl. tochinoides semblent confirmer, selon laquelle les ovarioles fonctionnent dans un ordre constant, il est possible de déterminer l'âge physiologique d'une femelle jusqu'à la septième ovulation incluse, soit, approximativement, jusqu'à l'âge de 78 à 80 jours.

La durée moyenne de vie des femelles de Gl. tachinoides, dans la nature, pouvanţ être estimée à 90 jours environ, cette méthode devrait permettre d'estimer l'âge d'une population avec une bonne précision.

.II ne semble pas, aınsı que l'affirme SAUNDERS, qu'après la première ovulation, l'utérus contienne toujours soit un œuf, soit une larve aux stades I, II ou II!. II semble bien, au contraire, qu'une période de repos d'1 à 2 jours intervienne après chacune des pontes larvaires.

Ces constatations demandent toutefois à être confirmées sur le terrain par l'étude des ovaires et de l'utérus de femelles de Gl. tachinoides capturées dans la nature, et de femelles écloses au laboratoire, marquées, lâchées dans la nature, puis recapturées.

\section{Institut d'élevage ef de Médecine vétérinaire} des IPays Tropicoux.

\section{SUMMARY}

\section{Oogenesis cycle in Glossina tachinoides West. female and determination of the physiological age}

A study of the genital and oogenesis cycle of Gl. tachinoides West. female which have been reared in laboratory af $25^{\circ} \mathrm{C}$ and $70 \mathrm{p}, 100$ relativ humidity and fecundated at 3 days of age, was carried out.

Anatomy and morphology of the female reproductive organs in this species are not very different from those which have already been described in other species of the same genus.

The oogenesis cycle is almost identical with the one of the species which have 
been still studied by various authors. A method and a table for the delermination of the physiological age of the females of Gl. tochinojdes are proposed.

By the method which is described, it is possible to asses the age of a female up to about 80 th day.

\section{RESUMEN}

Cielo de la oogenesis en las hembras de Glossina tachinoides West. y determinación de la edad fisiológica.

Se estudiaron el aparato genital y el ciclo de la oogenesis de las hembras de Gl. tachinoides West. criadas en el laboratorio a $25^{\circ} \mathrm{C}$ y 70 por 100 de relativa humedad, $y$ fecundadas a tres dias de edad.

La analomia y la morfología de los órganos reproductores de las hembras de esta especie son muy poco diferentes de las ya descritas en otras especies del mismo género.

El ciclo de la oogenesis es tambien casi idéntico al de las especies ya estudiadas por varios autores.

Se proponen un método y un cuadro de determinación de la edad fisialógica de las hembras de Gl. tochinoides El dicho método permite evaluar la edad de una hembra hasta ef $80^{\circ}$ dia poco más o menos.

\section{BIBLIOGRAPHIE}

BURSELL (E.) et JACKSON (C. H. N.). - Notes on the choriothete and milk gland of Glossina and hippobosca (Diptero). Prac. Roy. Ent. Soc. London, série A, 1957, 32 : 30-34.

BURTT (E.) et JACKSON (C. H. N.). - Illustration of tsetse larvae. Bull. Ent. Res. 1951, 41 (3) : 523-527.

BUXTON (P. A.). - The natural history of tsetse flies, 1955. Lewis et Cie ed. London.

BUXTON (P. A.) et LEWIS (D. J.). - Climate and tsetse flles: Laboratory studies upon Glossino submorsitans and tachinoides. Phil. trans. R. Soc. of London, série B, 1934, 224 : 175-240.

CHALLIER (A.). - Observations sur l'ovulation chez Glossino polpalis gambiensis Vand. 1949. Bull. Soc. Potho. exot., 1964, 57 (5) : 985-991.

CHALLIER (A.). - Amélioration de la méthode de détermination de l'âge physiologique des glossines. Etudes faites sur Glossina palpalis gambiensis Vand. 1949. Bull. Soc. Path. exot., 196558 (2) : 250-59.

DETINOVA (T. S.). - Méthodes à appliquer pour classer par groupes d'âge les diptères présentant une importance médicale, notamment certains vecteurs du paludisme. Org. mond. Santé. Série Monographie., 1963, $47: 220$.

GRUVEL (J.) et BALIS (J.). - Essai d'élevage de Glossina tachinoides West. au laboratoire. Rev. El. Med. vet. Pays trop., 1966, 19 (1) : 21-28.

HOFFMANN (R.). - Zur Fortpflanzungsbiologie und zur intrauterinen Entwicklung von Glossino palpalis. Acto trop., 1954, 11 (1) : 2-57.

ITARD (J.) et MAILLOT (L.). - Elevage en France, de Glossina morsitons morsitans West., ef Gl. tachinoides West. C. R. Acad. Sci., 1965, $261:$ :5626-8.

ITARD (J.) ef MAILLOT (L.). - Notes sur un élevage de Glossines (Diptera Muscidae) entrepris, à partir de pupes expédiées d'Afrique, à Maisons-Alfort (France). Rev. El. Med. vet. Pays, trop., 1966, 19 (1) : 29-44.

MINCHIN (E. A.). - Rapport on the anatomy of the tsetse fly (Glossina palpalis). Proc. Roy, Soc. London, série B, 1905, 76 : 531547.

ROUBAUD (E.). - La Glossina polpalis R.Des. : sa biologie, son rôle dans l'étiologie des trypanosomiases. Rapport de la mission 
d'étude de la maladie du sommeil au Congo françois (1906-1908). Masson éd. Paris.

SAUNDERS (D. S.). - Ovaries of Glossina morsitans. Noture, London, 1960, 185 : 121122.

SAUNDERS (D. S.). - Determination of physiological age for female Glossina morsitans. Nature, London, 1960, $186: 651$.

SAUNDERS (D. S.). - The ovulation cycle in Glossina morsitans West. (Diptera-Muscidae) and a possible method of age determination for female tsetse flies by the examination of their ovaries. Trans. $R$. ent. Soc. London, 1960, 112 (9) : 221-238.

SAUNDERS (D. S.). - Studies on ovarian deve- lopment in tsetse flies: (Glossino Dipfera). Parasitology, 1961, 51 : 545-564.

SAUNDERS (D. S.). - Age determination for female tsetse flies and the age compositions of samples Glossino pallidipes Aust., Gl. palpalis fuscipes Newst. and GI. brevipalpis Newst. Buil. ent. Res., 1962, 53 : 579-595. STUHLMAN (F.). - Beitrầge zur Kenntnis der Tseisefliege (Glossina) fusca. Gl. tachinoides). Arb. Gesundh Amt. Berl., 1907, $26: 301-383$.

VATTIER (G.). - Etude de caractères morphologiques ef anatomiques en relation avec l'âge physiologique des femelles de Glossines. Cahiers ORSTOM. Ent. Méd., n० 2,1964: 2-53. 